\title{
Antidepressant medication use and breast cancer risk
}

\author{
Karen J. Wernli, PhD ${ }^{1}$, John M. Hampton, MS $^{2}$, Amy Trentham-Dietz, PhD' , and Polly A. \\ Newcomb, PhD $^{1,2}$ \\ ${ }^{1}$ Program in Cancer Prevention, Fred Hutchinson Cancer Research Center, Seattle, WA 98109 \\ 2University of Wisconsin Paul P. Carbone Comprehensive Cancer Center, Madison, WI 53726
}

\begin{abstract}
Purpose-Most epidemiologic studies have detected no association between prior use of antidepressants medications and breast cancer risk. Despite the uniform conclusion, there is a continuous rise in the proportion of women using antidepressants lending support to further monitoring of disease effects.
\end{abstract}

Methods-We conducted a population-based case-control study among 2,908 incident breast cancer cases diagnosed from 2003 to 2006, and 2,927 control women from Wisconsin.

Associations between antidepressant use and breast cancer risk were evaluated using multivariable logistic regression.

Results-The association between use of antidepressant medications and breast cancer risk was null ( $\mathrm{OR}=0.89,95 \%$ CI $0.78-1.01)$. When stratified by type of antidepressant, use of selectiveserotonin reuptake inhibitors (SSRI) resulted in a similar risk overall $(\mathrm{OR}=0.85,95 \%$ CI 0.72 1.00 ) and among former and currents users. There were no associations between other types of antidepressant classes and breast cancer risk. In assessing risks among the five most commonly used antidepressants, we detected no association with fluoxetine, sertraline, venlafaxine or buproprion hydrochloride. There was a reduction in risk of $36 \%$ (OR=0.64, 95\% CI 0.45-0.92) among paroxetine hydrochloride. When stratified by body mass index, there was a reduction in risk associated with antidepressant users who were not overweight ( $\mathrm{OR}=0.73,95 \%$ CI $0.60-0.90)$, but this association was null in overweight women (p-interaction=0.04).

Conclusions-Surveillance of health risks associated with antidepressant medications continues to be of public health importance, though these medications are not likely to be associated with breast cancer risk.

\section{Keywords}

antidepressant medication; breast cancer; incidence; paroxetine hydrochloride; selective serotonin reuptake inhibitors

\section{INTRODUCTION}

There is some biologic evidence that antidepressants are related to cancer risk. Certain antidepressants promote mammary tumor growth in mouse models.1 Further, administration of antidepressants has been associated with acute increases in prolactin levels in woman, 2 and higher serum prolactin levels have been associated with increased breast cancer risk.3 Nevertheless, epidemiologic data have not supported these hypotheses. 4

Correspondence to: Karen J. Wernli, Fred Hutchinson Cancer Research Center, 1100 Fairview Ave N, M4-B402, Seattle, WA 98109; email: kwernli@fhcrc.org; tel 206.667.6228; fax 206.667.7850. 
Although there is uniformity in the overall null conclusion, a plaguing issue in prior research has been the definition of antidepressant exposure. Discrepancies in studies include the type of antidepressants used and introduction of new medications, the definition of minimum use for "ever" use, women who had used antidepressants for short- versus long-durations, and changes in prescription practices in classes of antidepressants utilized. $5^{-} 15$ Over the past 30 years, the most common class of antidepressant prescribed has changed from monoamine oxidase inhibitor (MAOI) and tricyclic antidepressants (TCA) to selective serotonin reuptake inhibitors (SSRI) and other reuptake inhibitors.16

Most importantly, though, has been the rapid increase in the prevalence of use of these psychotropic medications. Among US women, the prevalence of use has nearly doubled from $7.5 \%$ in 1988-1994 to $13.9 \%$ in 1999-2002, according to National Health and Nutrition Examination Survey (NHANES) data.16 Long-term surveillance of cancer risk among antidepressant users is imperative given the rising prevalence of use.

We evaluated the breast cancer risk associated with newer classes of antidepressants and longer-term use in a population-based case-control study among incident breast cancer cases and population controls in Wisconsin. Further, we sought to identify women who might be at increased or decreased risk due to age and obesity. This recent population based casecontrol study is representative of current US women's antidepressant use patterns.

\section{MATERIALS AND METHODS}

\section{Case Population}

Eligible case women aged 20-69 years were diagnosed with incident invasive breast cancer from 2003-2006 and reported to Wisconsin's statewide tumor registry. Eligible case subjects had a listed telephone number and driver's license verified by self-report. A total of 4,021 breast cancer cases were eligible for the study. We were not able to interview all cases due to inability to locate $(n=198)$, subject refusal $(n=747)$ or subject's death $(n=84)$. A total of 2,992 women were interviewed (overall response rate 74\%). Information collected from four cases was considered unreliable by the interviewers, leaving a total of 2,988 case women for analysis.

\section{Selection of Controls}

Controls were randomly selected from the community using a list of licensed drivers. All eligible controls also had a publicly available telephone number. Controls were frequency matched to the cases within 5-year age stratum. We identified 4,500 eligible women, but were not able to interview all women due to inability to locate $(n=327)$, subject refusal $(n=1,152)$ or subject's death $(n=16)$. A total of 3,005 women were interviewed (overall response rate $67 \%$ ). Information collected from one control was considered unreliable by the interviewer, leaving a total of 3,004 controls for analysis.

\section{Data collection}

All potential study participants were mailed a letter introducing them to the study before they were contacted on the telephone. All study subjects provided informed consent. A trained interviewer administered a 30-minute telephone interview which elicited information on current and past use of antidepressant medications. Women were asked the name of the medication, how frequently they took the medication, the start and stop years, and how long in total that they took the medication. Reasons for last use of the antidepressant were also ascertained in a subsample of women. Women were also asked about demographic characteristics, reproductive history, personal and family cancer history, physical activity, smoking, and alcohol consumption. 
For each case, a reference date was defined as the registry-supplied date of invasive breast cancer diagnosis. For comparability, the control subjects, who were interviewed contemporaneously with cases, were assigned an individual reference date. A reference date for each control was calculated based the normal distribution of time from diagnosis to interview among the cases based on 5-year age-groups. Establishing a reference date was done to maintain comparability between cases and controls, and to maintain interviewer blinding to case-control disease status. For example, if the average time to interview was approximately 9 months for cases aged 50-54 years, then the control reference data was set at 9 months prior to the interview. Reference age was defined as the woman's age at the reference date. Only exposures that occurred at least a year prior to the assigned reference date were included in analyses.

This study was approved by the Institutional Review Board (IRB) at the University of Wisconsin, Paul P. Carbone Comprehensive Cancer Center.

\section{Statistical Analysis}

We excluded women from the analysis who did not respond to the questions regarding antidepressant use ( 80 cases and 77 controls), so the final sample included 2,908 breast cancer cases and 2,927 controls.

Ever use of antidepressant medication was defined as use for three months or more. A woman was considered a current user if she reported use within 12 months of the reference date including an episode at least three months in duration. Former use was defined as use for at least three months duration, but prior to the 12 months preceding the reference date. A woman was defined as a never user if she responded that she had never used or used antidepressants for less than three months. In general, former users ceased their antidepressant medication about 6-7 years before the reference date.

Duration of use was categorized as never, $<2,2-4,5-9$, and $>10$ years. We evaluated the risk of breast cancer associated with antidepressant use overall and also by class of antidepressant. Classes of antidepressants included selective serotonin receptor inhibitors (SSRI), tricyclic antidepressants (TCA), norepinephrine-dopamine reuptake inhibitors (NDRI), and serotonin-norepinephrine reuptake inhibitor (SNRI). There were too few women who took monoamine oxidase inhibitors (MAOIs) to evaluate separately ( $<10$ women). Women who reported the use of more than one type of antidepressant were counted in the overall main effect of antidepressant use, but excluded from class-specific analyses. We also calculated risk of breast cancer with the five most commonly used antidepressants.

Women were classified as postmenopausal if they reported natural menopausal prior to the reference date. Women who had at least one ovary and had a hysterectomy were classified as premenopausal if their reference age was in the first decile of age at natural menopause among the controls, and as postmenopausal if their reference age was in the highest decile for age at natural menopause in the control group. A woman's menopausal status was considered unknown if she had undergone a hysterectomy without bilateral oophorectomy at an intermediate age (second to ninth decile).

Odds ratios and 95 percent confidence intervals (CI) obtained from logistic regression models were used to evaluate relative risks utilizing SAS version 9.1 (SAS Institute, Inc, Carey, N.C.). 17 Models were adjusted for age at reference date, year of interview, parity, age at first live birth, body mass index at reference date, family history of breast cancer, menopausal status, age at menopause, history of mammography (annual mammogram each year in the last 5 years), and type of hormone use (none, estrogen-alone, estrogen plus 
progestin). Trends were evaluated by entering an ordinal term representing the category of antidepressant use duration into a regression model.

To test for interaction of antidepressant use with body mass index and age, a cross-product term was entered into the model, and the likelihood ratio test was used to test the difference between the full model and reduced model.

\section{RESULTS}

Case women were significantly more likely than control women to report a first-degree family history of breast cancer, having a higher education, being nulliparous, having a first born child after age 30 , having gained more $10 \mathrm{~kg}$ of weight since age 20 , and taking estrogen-progestin hormone therapy (Table 1).

Approximately $23 \%$ of control women had ever used antidepressants (Table 2). Overall, there was no association between use of antidepressant medications and breast cancer risk $(\mathrm{OR}=0.89,95 \% \mathrm{CI}$ 0.78-1.01). Relations among former and current users were similar to overall use. There was no dose-response relationship by duration of use (Table 2).

When stratified by type of antidepressant use, ever use of SSRI was not associated with breast cancer risk (OR=0.85, 95\% CI 0.72-1.00) (Table 2), which was similar among both former and currents users. There were no associations between other classes of antidepressant medications (i.e., TCA, NDRI or SNRI) use and breast cancer risk

The top five most commonly used drug preparations were fluoxetine hydrochloride, sertraline hydrochloride, paroxetine hydrochloride, venlafaxine hydrochloride, and buproprion hydrochloride (Table 3). There was a reduced risk of breast cancer associated with former users of fluoxetine hydrochloride, but no association among ever or current users. We detected a reduction in breast cancer risk associated with use of paroxetine hydrochloride $(\mathrm{OR}=0.64,95 \%$ CI $0.45-0.92)$, which was limited to current users. Further, there was a suggestion of a decreasing trend with increasing duration of use of paroxetine hydrochloride, though we lacked statistical power to detect a significant association (ptrend $=0.10$ ). There were no other associations according to any of the four other types of antidepressants and breast cancer risk.

We evaluated whether the risks of ever use of antidepressants and breast cancer risk might be modified by age or obesity (Table 4). There was no suggestion of a difference in risk by age for overall use, by type or duration. Among women who were not overweight (BMI< $25)$, there was a $27 \%$ reduction in breast cancer risk among ever users of antidepressants compared never users $(\mathrm{OR}=0.73,95 \%$ CI $0.60-0.90)$; on the contrary, women who were overweight had no reduction in risk of breast cancer associated with ever use of antidepressants (p-interaction=0.04) (Table 4). Both former and current users among not overweight women had reductions in risk. Among non-overweight women, those who had taken SSRI had 34\% reduction in risk compared to never users ( $\mathrm{OR}=0.66,95 \% \mathrm{CI} 0.51-$ $0.85)$; among women who were overweight, the association was null ( $\mathrm{OR}=1.02,95 \% \mathrm{CI}$ $0.82-1.26)(\mathrm{p}$-interaction $=0.01)$. There were no significant interactions with other classes of antidepressants or duration of use.

We conducted a sensitivity analysis to understand whether the diagnosis of depression versus the medical use of an antidepressant was associated with breast cancer risk. The results were stratified by four identified indications for use: 1) depression; 2) anxiety; 3) menstrual problems; and 4) other (e.g. pain). The majority of subjects used antidepressants for depression or anxiety. The overall odds ratios for ever use of antidepressants remained null in all four categories [depression $(\mathrm{OR}=0.81,95 \mathrm{CI} 0.66-1.01)$, anxiety $(\mathrm{OR}=0.70,95 \%$ 
CI 0.41-1.21), menstrual problems ( $\mathrm{OR}=1.50,95 \% \mathrm{CI} 0.70-3.37)$, and other $(\mathrm{OR}=0.88$, $95 \%$ CI $0.57-1.35)]$. These results suggest that confounding by indication was unlikely to be an issue.

\section{DISCUSSION}

Similar to other research, we detected no strong association between ever use of antidepressants and breast cancer risk.4 However, in some classes of antidepressants, breast cancer risk was reduced. There was a decreased risk of breast cancer associated with use of paroxetine hydrochloride and potentially fluoxetine. There is also evidence that antidepressant users who were not overweight had a decreased risk of breast cancer.

Most studies have detected relative risks ranging from $0.7-1.3$ for ever use of SSRIs. ${ }^{-} 11$, 13,15 Our finding for a modest 0.9 odds ratio is within the range of previous studies. One of the most recent and largest studies using the General Practice Research Databases in the UK assessed both SSRI and TCA use and breast cancer risk in a nested case-control study. 9 The cases were identified and reviewed through the medical record system, and the exposure was assessed using the drug database. Among 3,708 breast cancer cases and 20,000 controls, the use of SSRIs was not associated with breast cancer risk ( $\mathrm{OR}=0.98,95 \%$ CI 0.81-1.19). Among this study population, the prevalence of SSRI use in the controls was $4.4 \%$, similar to the prevalence reported in other study populations.7, 8 Approximately $12.2 \%$ of controls in our study have used a SSRI, which most likely reflects the rise in use nationally.

The reduction in breast cancer risk associated with paroxetine hydrochloride was unexpected. This medication was released to the market in 1992.18 Observational studies began to specifically analyze the association of paroxetine hydrochloride and breast cancer risk after an initial report of a seven-fold increased risk.6 Subsequent evaluations have detected both modest decreased risks $7^{-} 9$ or no association with breast cancer.10, 15 FultonKehoe investigated the number of paroxetine prescriptions filled among women living in western Washington and detected nearly a $50 \%$ reduction in breast cancer risk $(\mathrm{OR}=0.52$, 95\% CI $0.21-1.31$ ) among women prescribed 11 or more prescriptions compared to nonusers. 8 In our analysis, we did detect both a decrease in risk and a suggestion of doseresponse relationship with paroxetine hydrochloride use, which warrants further attention.

We had investigated the interaction between antidepressants and body weight one year prior to the reference date given that body mass can affect the metabolism of medications. In our analysis, women who were not overweight had a reduced risk of breast cancer associated with antidepressant use, while women who were overweight showed no increased or decreased risk of breast cancer. To our knowledge, this finding has not been reported previously and should be further evaluated within sufficiently powered studies with available data on body weight. We detected no differences by age.

One issue to be addressed is whether depression is a risk factor for breast cancer and therefore, may be a confounder in the relationship between antidepressant use and cancer risk. Depression has been associated with overall cancer incidence 19, 20 and breast cancer. 21 In our stratified analysis of the indication of use, we did not detect any differences in the overall association of antidepressant use and breast cancer risk, suggesting that confounding by indication was unlikely to be an issue in these results.

As in all studies, the assessment of exposure is important. We relied on self-report of antidepressant medication for this analysis. Some studies have utilized pharmacy database records9, 10,14 and others have relied on self-report.6, $7,11,15$ One difficulty with pharmacy database-linkage studies is the paucity of information on other potential confounding factors, such as parity or mammography use. Overall recall of antidepressant 
medications appears to be moderate. In a validation study of self-report of antidepressants and pharmacy records, the sensitivity of recall was 56\% in cases and 58\% in controls within a two year period of use. 22 The specificity of recall was nearly $100 \%$. The median length of time from cessation of antidepressant use to reference date was 7 and 6 years for cases and controls, respectively. This suggests that our study might have under-ascertained exposure, which is likely to be non-differential, and results could be biased towards the null, which reflects our own current findings.

While epidemiologic evidence continues to suggest no strong overall breast cancer risk associated with antidepressant use, further studies should continue to investigate this issue. Women will continue to be using antidepressants for longer periods of time and for a variety of medical conditions. The introduction of new antidepressants suggests that surveillance of health risks associated with these medications is of public health importance.

\section{Key Points}

- Overall use of antidepressants is not associated with breast cancer risk.

- Use of paroxetine hydrochloride may be associated with a reduced risk of breast cancer.

- Women who are normal weight have a reduced risk of breast cancer associated with use of antidepressant medications.

- Women who are overweight have no reduction in risk of breast cancer with use of antidepressant medications.

- Surveillance of health risks associated with antidepressant medications continues to be of public health importance.

\section{Acknowledgments}

The authors are grateful to Laura Stephenson and the staff of the Wisconsin Cancer Reporting System, Dr. Jane McElroy and Hazel Nichols for data analysis support, and Julie McGregor along with the study interviewers and programmers for assistance with data collection. We acknowledge the support of Dr. Katia Ronay on the assessment of antidepressants. We are especially grateful to the study participants, whose generosity made this research possible.

Financial support: This research was funded by National Institute of Health grants R01 CA47147 and CA47305. The authors have no other conflicts to report.

\section{REFERENCES}

1. Brandes LJ, Arron RJ, Bogdanovic RP, et al. Stimulation of malignant growth in rodents by antidepressant drugs at clinically relevant doses. Cancer Res. 1992; 52:3796-3800. [PubMed: 1617649]

2. Cowen PJ, Sargent PA. Changes in plasma prolactin during SSRI treatment: evidence for a delayed increase in 5-HT neurotransmission. J Psychopharmacol. 1997; 11:345-348. [PubMed: 9443523]

3. Tworoger SS, Eliassen AH, Rosner B, Sluss P, Hankinson SE. Plasma prolactin concentrations and risk of postmenopausal breast cancer. Cancer Res. 2004; 64:6814-6819. [PubMed: 15375001]

4. Coogan PF. Review of the epidemiological literature on antidepressant use and breast cancer risk. Expert Rev Neurother. 2006; 6:1363-1374. [PubMed: 17009923]

5. Kelly JP, Rosenberg L, Palmer JR, et al. Risk of breast cancer according to use of antidepressants, phenothiazines, and antihistamines. Am J Epidemiol. 1999; 150:861-868. [PubMed: 10522657]

6. Cotterchio M, Kreiger N, Darlington G, Steingart A. Antidepressant medication use and breast cancer risk. Am J Epidemiol. 2000; 151:951-957. [PubMed: 10853633] 
7. Chien C, Li CI, Heckbert SR, Malone KE, Boudreau DM, Daling JR. Antidepressant use and breast cancer risk. Breast Cancer Res Treat. 2006; 95:131-140. [PubMed: 16322894]

8. Fulton-Kehoe D, Rossing MA, Rutter C, Mandelson MT, Weiss NS. Use of antidepressant medications in relation to the incidence of breast cancer. Br J Cancer. 2006; 94:1071-1078. [PubMed: 16523201]

9. Gonzalez-Perez A, Garcia Rodriguez LA. Breast cancer risk among users of antidepressant medications. Epidemiology. 2005; 16:101-105. [PubMed: 15613952]

10. Haque R, Enger SM, Chen W, Petitti DB. Breast cancer risk in a large cohort of female antidepressant medication users. Cancer Lett. 2005; 221:61-65. [PubMed: 15797628]

11. Moorman PG, Grubber JM, Millikan RC, Newman B. Antidepressant medications and their association with invasive breast cancer and carcinoma in situ of the breast. Epidemiology. 2003; 14:307-314. [PubMed: 12859031]

12. Sharpe CR, Collet JP, Belzile E, Hanley JA, Boivin JF. The effects of tricyclic antidepressants on breast cancer risk. Br J Cancer. 2002; 86:92-97. [PubMed: 11857018]

13. Steingart A, Cotterchio M, Kreiger N, Sloan M. Antidepressant medication use and breast cancer risk: a case-control study. Int J Epidemiol. 2003; 32:961-966. [PubMed: 14681256]

14. Wang PS, Walker AM, Tsuang MT, Orav EJ, Levin R, Avorn J. Antidepressant use and the risk of breast cancer: a non-association. J Clin Epidemiol. 2001; 54:728-734. [PubMed: 11438414]

15. Coogan PF, Palmer JR, Strom BL, Rosenberg L. Use of selective serotonin reuptake inhibitors and the risk of breast cancer. Am J Epidemiol. 2005; 162:835-838. [PubMed: 16177141]

16. Paulose-Ram R, Safran MA, Jonas BS, Gu Q, Orwig D. Trends in psychotropic medication among US adults. Pharmacoepidemiol Drug Saf. 2007; 16:560-570. [PubMed: 17286304]

17. Breslow NE, Day NE. Statistical methods in cancer research. Volume I - The analysis of casecontrol studies. IARC Scientific Publications (Lyon). 1980:5-338.

18. Mojtabai R. Increase in antidepressant medication in the US adult population between 1990 and 2003. Psychother Psychosom. 2008; 77:83-92. [PubMed: 18230941]

19. Linkins RW, Comstock GW. Depressed mood and development of cancer. Am J Epidemiol. 1990; 132:962-972. [PubMed: 2239911]

20. Penninx BW, Guralnik JM, Pahor M, et al. Chronically depressed mood and cancer risk in older persons. J Natl Cancer Inst. 1998; 90:1888-1893. [PubMed: 9862626]

21. Gallo JJ, Armenian HK, Ford DE, Eaton WW, Khachaturian AS. Major depression and cancer: the 13-year follow-up of the Baltimore epidemiologic catchment area sample (United States). Cancer Causes Control. 2000; 11:751-758. [PubMed: 11065012]

22. Boudreau DM, Daling JR, Malone KE, Gardner JS, Blough DK, Heckbert SR. A validation study of patient interview data and pharmacy records for antihypertensive, statin, and antidepressant medication use among older women. Am J Epidemiol. 2004; 159:308-317. [PubMed: 14742292] 


\section{Table 1}

Percentage of selected demographic characteristics for breast cancer cases and population-based controls.

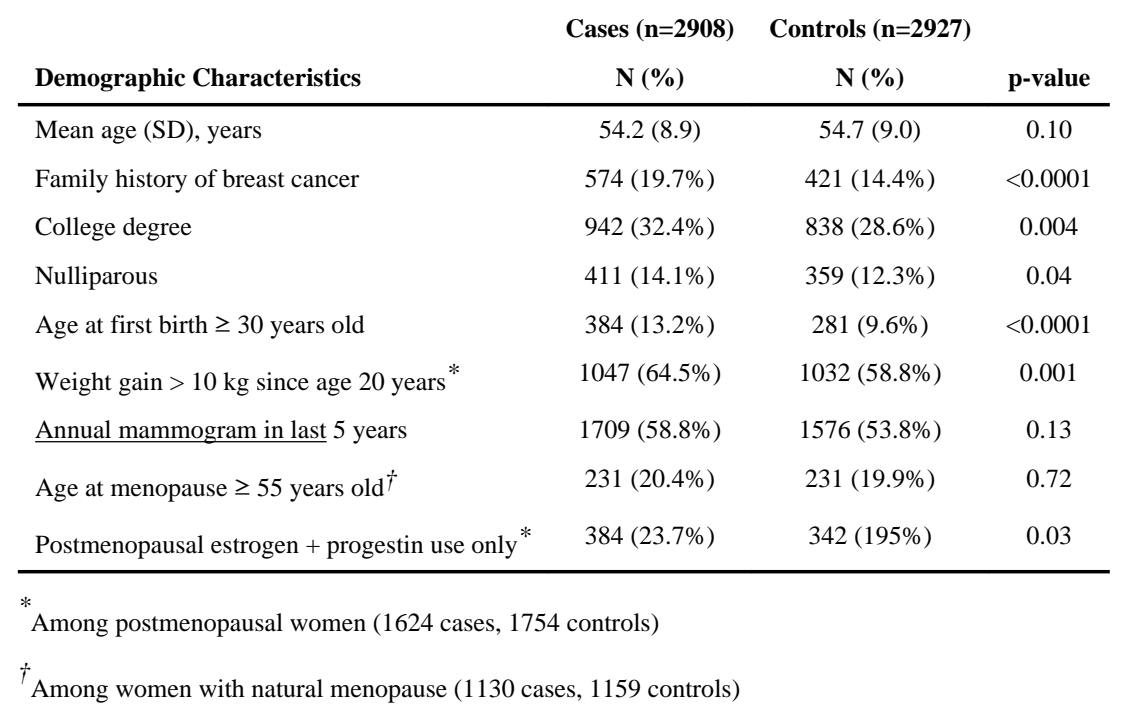


Table 2

Risk of breast cancer according to duration and recency of antidepressant use overall and by class.

\begin{tabular}{lccc} 
& Cases (n=2908) & Controls (n=2927) \\
Antidepressant use & $\mathbf{N}$ & $\mathbf{N}$ & OR (95\% CI) \\
\hline $\begin{array}{l}\text { Never use } \\
\text { Any use }\end{array}$ & 2292 & 2262 & 1.00 (Referent) \\
Ever use & & & \\
$\quad$ Former & 616 & 665 & $0.89(0.78-1.01)$ \\
Current & 166 & 200 & $0.82(0.66-1.01)$ \\
Duration (years) & 450 & 465 & $0.92(0.80-1.07)$ \\
$<2$ & & & \\
$2-4$ & 211 & 247 & $0.83(0.68-1.02)$ \\
$5-9$ & 180 & 169 & $1.00(0.80-1.25)$ \\
$>10$ & 124 & 139 & $0.87(0.67-1.12)$ \\
& 101 & 112 & $0.87(0.66-1.15)$
\end{tabular}

Selective Serotonin Reuptake Inhibitors (SSRI)

Ever use

Former

Current

Duration (years)

$<2$

2-4

5-9

$>10$

Tricyclic Antidepressant (TCA)

Ever use $\quad 48$

Former

Current

Duration (years)

$\begin{array}{cccc}<2 & 14 & 18 & 0.86(0.42-1.75) \\ 2-4 & 11 & 9 & 1.10(0.45-2.72) \\ 5-9 & 9 & 9 & 1.04(0.41-2.68) \\ >10 & 14 & 17 & 0.78(0.38-1.60)\end{array}$

Norepinephrine-dopamine reuptake inhibitors (NDRI)

Ever use

Former

48

Current

14

34

Duration (years)

$<2$

$2-4$

5-9

$>10$

23

15

8

2
357

100

257

128

109

71

49

$53 \quad 0.90(0.60-1.35)$

20

33

$0.93(0.48-1.79)$

0.89 (0.54-1.48)

$0.86(0.42-1.75)$

$1.10(0.45-2.72)$

$0.78(0.38-1.60)$

$0.92(0.62-1.38)$

0.73 (0.37-1.46)

$1.04(0.64-1.71)$

$0.76(0.43-1.32)$

$1.65(0.71-3.83)$

0.88 (0.34-2.25)

$0.61(0.10-3.70)$

Serotonin-norepinephrine reuptake inhibitor (SNRI) 


\begin{tabular}{lccc} 
& Cases $(\mathbf{n}=\mathbf{2 9 0 8})$ & Controls $(\mathbf{n = 2 9 2 7})$ & \\
Antidepressant use & $\mathbf{N}$ & $\mathbf{N}$ & OR $(\mathbf{9 5 \%} \mathbf{C I})^{*}$ \\
\hline Ever use & 37 & 31 & $1.18(0.72-1.92)$ \\
Former & 6 & 5 & $1.14(0.34-3.83)$ \\
$\quad$ Current & 31 & 26 & $1.18(0.70-2.02)$ \\
Duration (years) & & & \\
$<2$ & 14 & 14 & $0.95(0.45-2.03)$ \\
$2-4$ & 12 & 10 & $1.27(0.54-2.98)$ \\
$5-9$ & 10 & 7 & $1.37(0.52-3.65)$ \\
$>10$ & 1 & 0 & - \\
\hline
\end{tabular}

* Adjusted for age, year of interview, parity, age at first live birth, family history of breast cancer, body mass index, menopausal status, age at menopause, mammography, and type of hormone use. 
Table 3

Risk of breast cancer according to duration and recency of the five most commonly used antidepressant.

\begin{tabular}{|c|c|c|c|}
\hline Antidepressant use & $\begin{array}{c}\text { Cases } \\
\mathbf{N}\end{array}$ & $\begin{array}{c}\text { Controls } \\
\mathbf{N}\end{array}$ & OR $(95 \% \mathrm{CI})^{*}$ \\
\hline Never use & 2296 & 2265 & 1.0 (Referent) \\
\hline \multicolumn{4}{|c|}{ Fluoxetine hydrochloride } \\
\hline Ever use & 106 & 114 & $0.88(0.67-1.16)$ \\
\hline Former & 24 & 48 & $0.47(0.28-0.77)$ \\
\hline Current & 82 & 66 & $1.18(0.85-1.67)$ \\
\hline \multicolumn{4}{|l|}{ Duration (years) } \\
\hline$<2$ & 30 & 46 & $0.60(0.38-0.97)$ \\
\hline $2-4$ & 23 & 24 & $0.95(0.53-1.71)$ \\
\hline $5-9$ & 22 & 20 & $1.02(0.55-1.90)$ \\
\hline$>10$ & 31 & 24 & $1.22(0.71-2.11)$ \\
\hline
\end{tabular}

Sertraline hydrochloride

$\begin{array}{cccc}\text { Ever use } & 94 & 90 & 0.98(0.73-1.33) \\ \text { Former } & 29 & 28 & 1.02(0.60-1.73) \\ \text { Current } & 65 & 62 & 0.97(0.67-1.38)\end{array}$

Duration (years)

$\begin{array}{lccc}<2 & 41 & 41 & 0.97(0.62-1.51) \\ 2-4 & 34 & 20 & 1.57(0.90-2.76) \\ 5-9 & 14 & 18 & 0.69(0.34-1.41) \\ >10 & 5 & 11 & 0.43(0.15-1.25)\end{array}$

Paroxetine hydrochloride

$\begin{array}{lccc}\text { Ever use } & 52 & 80 & 0.64(0.45-0.92) \\ \text { Former } & 17 & 19 & 0.89(0.45-1.74) \\ \text { Current } & 35 & 61 & 0.57(0.37-0.86) \\ \text { Duration (years) } & & & \\ <2 & 23 & 26 & 0.93(0.52-1.65) \\ 2-4 & 18 & 30 & 0.54(0.30-0.98) \\ 5-9 & 9 & 17 & 0.53(0.24-1.21) \\ >10 & 2 & 7 & 0.31(0.06-1.51)\end{array}$

Venlafaxine hydrochloride

$\begin{array}{cccc}\text { Ever use } & 30 & 35 & 0.78(0.47-1.29) \\ \text { Former } & 5 & 5 & 0.94(0.27-3.30) \\ \text { Current } & 25 & 30 & 0.75(0.44-1.30)\end{array}$

Duration (years)

$\begin{array}{cccc}<2 & 9 & 11 & 0.74(0.30-1.83) \\ 2-4 & 15 & 18 & 0.75(0.38-1.51) \\ 5-9 & 5 & 5 & 0.94(0.27-3.32) \\ >10 & 1 & 1 & 0.94(0.06-15.11)\end{array}$

Bupropion hydrochloride 


\begin{tabular}{lccc} 
& Cases & Controls & \\
Antidepressant use & $\mathbf{N}$ & $\mathbf{N}$ & OR $(\mathbf{9 5 \%} \mathbf{C I})^{*}$ \\
\hline Ever use & 53 & 48 & $1.09(0.73-1.63)$ \\
$\quad$ Former & 16 & 16 & $1.02(0.51-2.06)$ \\
Current & 37 & 32 & $1.12(0.69-1.83)$ \\
Duration (years) & & & \\
$<2$ & 26 & 23 & $1.11(0.63-1.97)$ \\
$2-4$ & 16 & 10 & $1.54(0.69-3.45)$ \\
$5-9$ & 9 & 12 & $0.79(0.33-1.90)$ \\
$>10$ & 2 & 3 & $0.61(0.10-3.70)$ \\
\hline
\end{tabular}

* Adjusted for age, year of interview, parity, age at first live birth, family history of breast cancer, body mass index, menopausal status, age at menopause, mammography, and type of hormone use. 


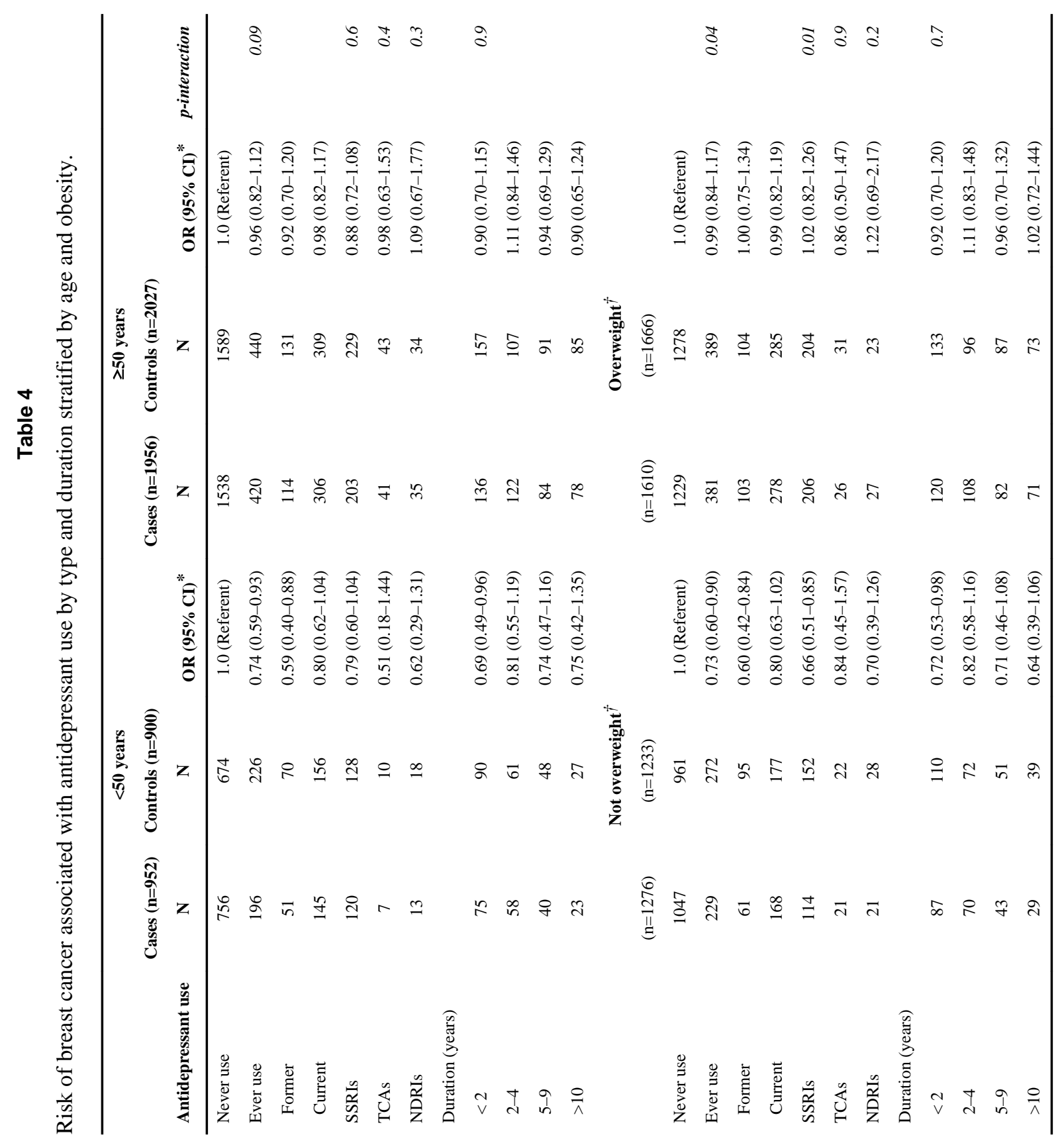


